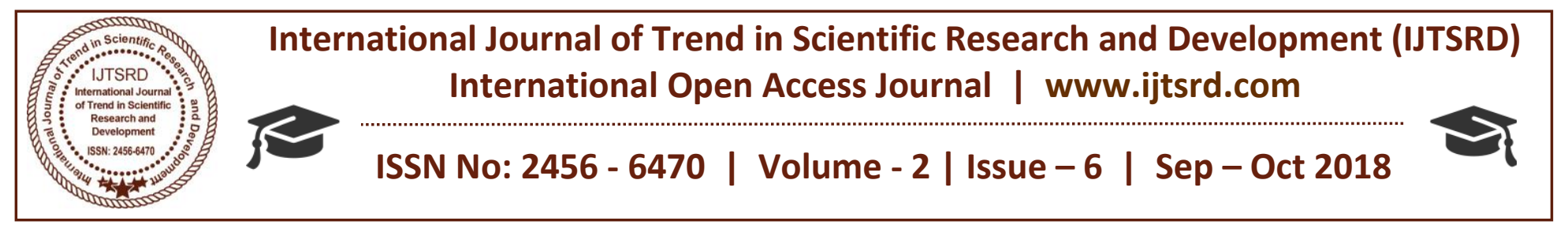

\title{
Mechanical Low Backache
}

\author{
Dr. Madhuri Paikera ${ }^{1}$, Laxmi Barve ${ }^{1}$, Subhra Dubey ${ }^{2}$ \\ Physiotherapist \\ ${ }^{1}$ Apollo College of Physiotherapy, Durg, Chattishgarh, India \\ ${ }^{2}$ Pt.JNM. Medical College Raipur Chattishgarh, India
}

\section{ABSTRACT}

Back pain is one of the most common patient complaints brought forth to physicians. Mechanical back pain accounts for $97 \%$ of cases, arising from spinal structures such as bone, ligaments, discs, joints, nerves, and meninges. Acute back pain in the absence of progressive neurologic deficits and other underlying non mechanical causes may be treated conservatively, with specific emphasis on maintaining activity levels and function. Mechanical back pain persisting for more than 4 to 6 weeks may warrant further diagnostic testing and imaging. Common causes of mechanical back pain include spinal stenosis, herniated discs, zygapophysial joint pain, discogenic pain, vertebral fractures, sacroiliac joint pain, and myofascial pain. A wide variety of treatments are available, with different treatments specifically targeted toward different causes./ A balanced approach, which takes into account patient psychosocial factors and incorporates multidisciplinary care, increases the likelihood of success from back pain interventions. The majority of people will experience mechanical low-back pain sometime in their lives. Mechanical low-back pain can be defined as pain resulting from excessive or prolonged posture issues, such as too much sitting or standing. Pain can also be caused from overuse or muscle strain, or it could be pain secondary to injury or deformity relating to the spinal structures, such as bone, ligaments, discs, joints, nerves, and meninges. Mechanical back pain persisting for more than 4 to 6 weeks may require diagnostic testing and imaging. Nonetheless, treatments are available, including physical therapy, with different programs specifically targeted toward different causes.

\section{KEY WORDS: mechanical LBA, Physiotherapy,} posture

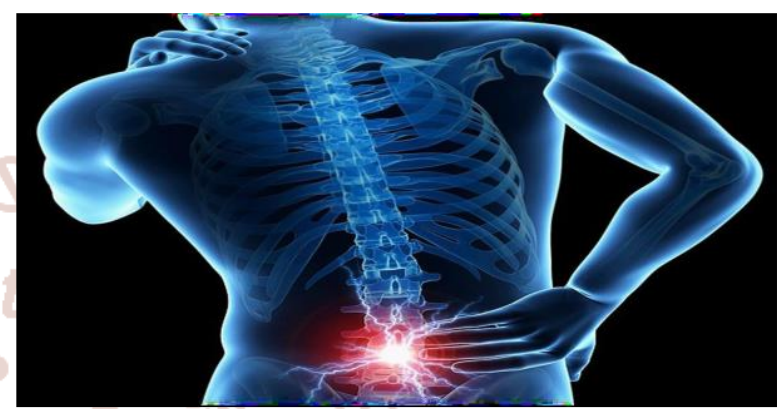

\section{BACKGROUND:-}

In last few years, we have been noticing a constant in the complaints of mechanical LBA due to bad ergonomics mostly seen in office workers without actually having any significant pathological issues.

As the center and axis of the human body, the Low back has a bear a variety of heavy loads during everyday movements and low back pain has been found. Thus, correct diagnosis and treatment of the underlying cause of LBA due to bad ergonomics is essential to maintaining the quality of life.

Its concluded that mechanical LBA, a disease of the axis of human body, has a critical influence not only on quality of life, but on the life of the elderly in an aged society.

Mechanical LBA is not a life-threatening illness.

Acute Mechanical Back pain is a common medical problem now a day's it's also known as Acute Low back pain, lumbago, idiopathic low back pain, lumbosacral strain or sprain, or lumbar syndrome.

Acute means that it has lasted less than 4 to 6 weeks.

Mechanical means the source of the pain may be in the spinal joints, discs, vertebrae, or soft tissues. 


\section{FUNCTIONAL UNIT OF LOW BACK:-}

The spinal column is made up of 26 bones: 24 unique vertebrae plus the sacrum and coccyx (tail bone) at the end of the backbone. The Vertebrae seem to be chained together). The vertebrae include:

$>7$ cervical vertebrae which makes up the neck

12 thoracic vertebrae of the chest

$>5$ lumbar vertebra or the "lower back" - L1, L2, L3, L4 and L5

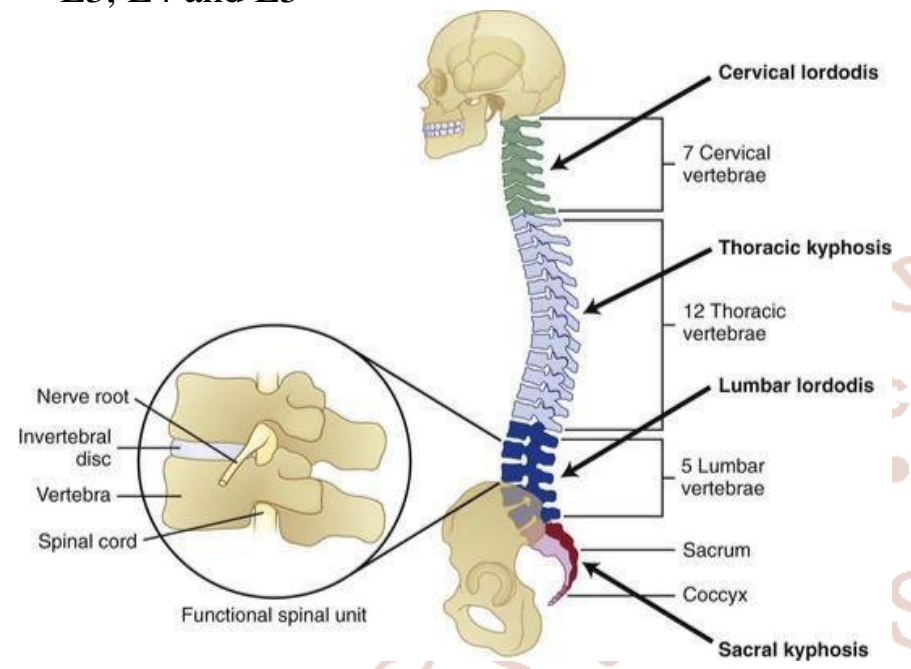

The Spinal Column
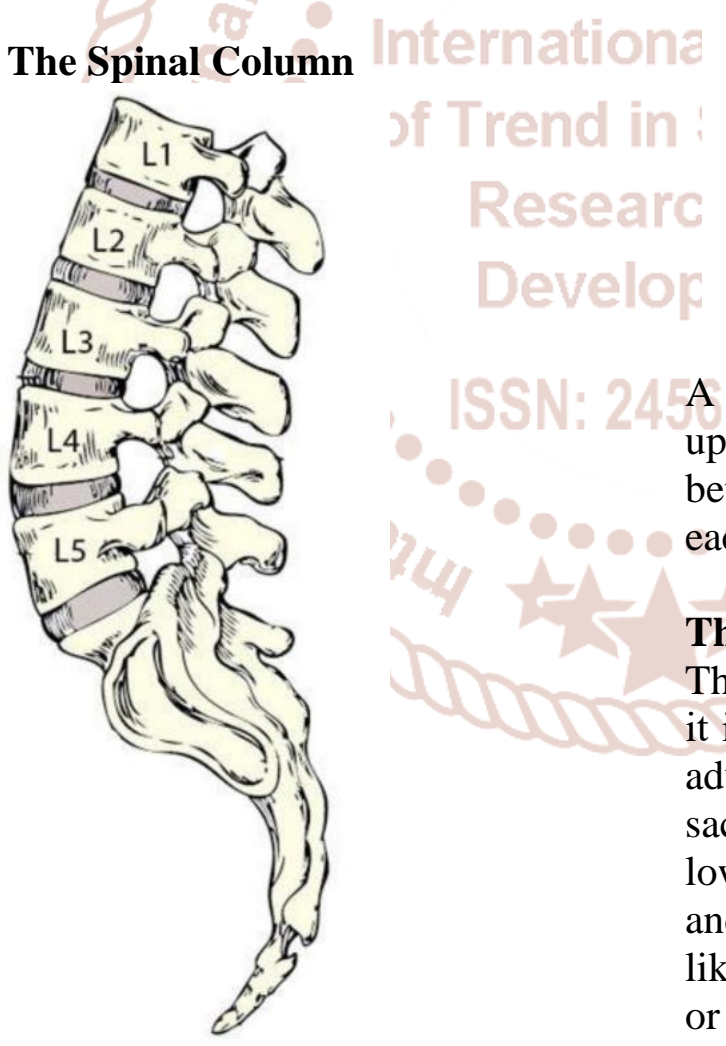

A spinal segment forms a functional unit and is made up of two adjacent vertebrae, the intervertebral disk between them, the two spinal nerves that exit from each side of the spinal cord, ligaments and muscles.

\section{The Sacrum}

The sacrum is the last segment of the spine. At birth, it is made of several vertebrae. By the time you're an adult these vertebrae have fused together to form the sacrum. The Sacrum is a large, triangular bone, in the lower part of the vertebral column and at the upper and back part of the pelvic cavity, where it is inserted like a wedge between the two hip bones; its upper part or base joins with the 5th lumbar vertebra by intervertebral fibro cartilage and at the bottom it joins with the coccyx or tailbone.

Bones, disks and facet joints of lumbar spine

The back can move in many different directions, it can stiffen as well as be supple. When looked at from the back, the spine appears to be straight, but looked at from the side you can see 2 curves which cause the back to have an "S" shape-it curves forward at the

\section{The Lumbar Spine}

The Lumbar spine consists of the vertebral body, posterior elements, intervertebral disks, and ligaments. The lumbar spine is made up of the five lumbar vertebrae located between the thoracic spine 
and the sacrum. This area is commonly called the "lower back". The lumbar vertebrae are the largest of the vertebrae because of their weight-bearing function supporting the torso and head.
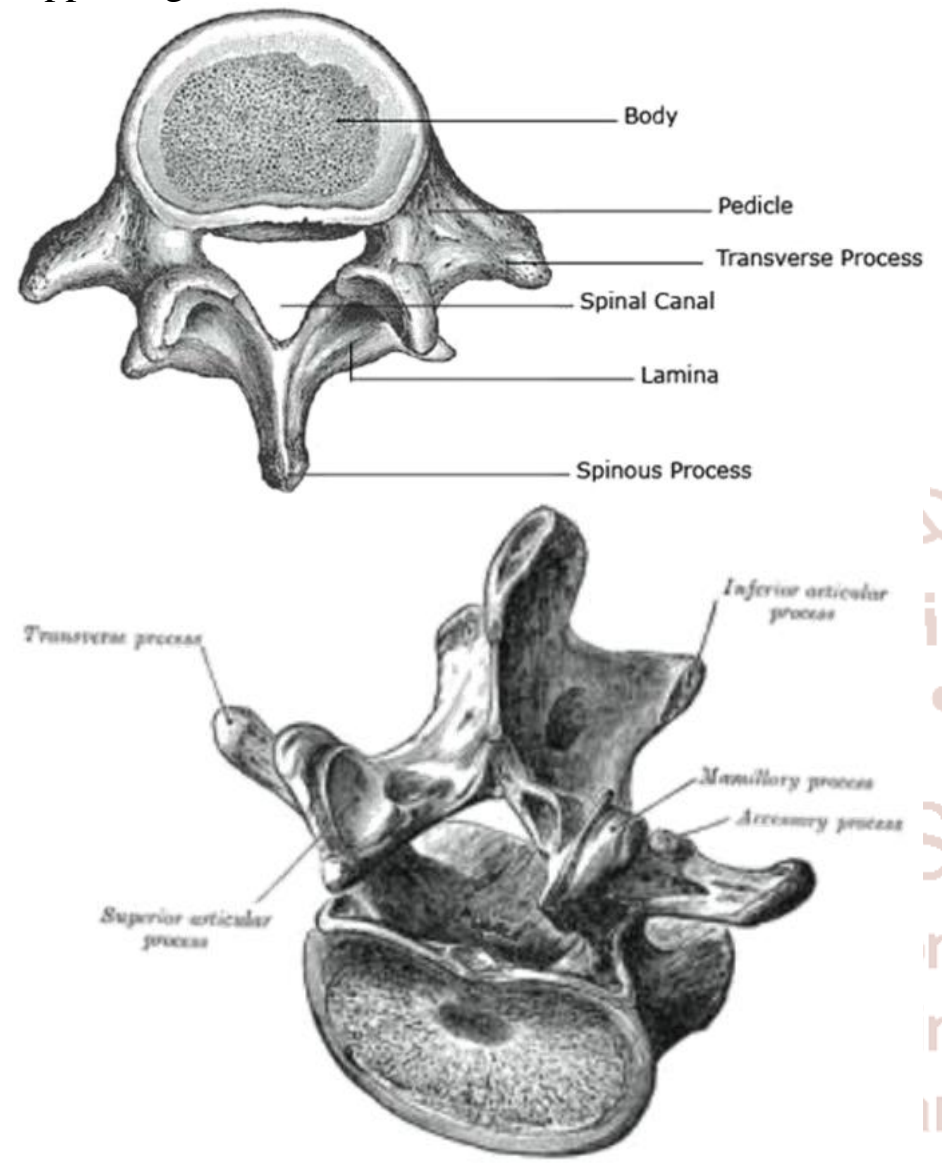

Labeled lumbar vertebra

The function of the structures of the lumbar spine are to protect and support the spinal cord and spinal nerves. The spinal nerves pass through a large hole (foramen) in the center of each vertebrae, which when lined up is called the spinal canal. The lumbar spinal nerves branch off the spinal cord at each level between the vertebrae. The joints - a joint is where two or more bones meet-between the vertebrae contain a disk (intervertebral disk) that acts as a shock absorber.

The vertebrae of the back are "linked" together by pedicles (lamina, transverse process, and spinous process) to form facet joints.

\section{Ligaments of the Back}

The function of ligaments is to attach bones to bones and give strength and stability to the back. Ligaments are strong, tough bands that are not very flexible. The vertebral bodies of the back are connected to each other by multiple ligaments which include:
$>$ posterior longitudinal ligaments

$>$ anterior longitudinal ligaments

$>$ intertransverse ligaments

$>$ interspinous ligaments

$>$ supraspinous ligaments

\section{Tendons of the Back}

Tendons are elastic tissues that connect muscles to bones.

\section{Muscles of the Back:-}

The muscles of the back can be divided into three groups - superficial, intermediate and deep:

$>$ Superficial - associated with movements of the shoulder.

$>$ Intermediate - associated with movements of the thoracic cage.

Deep - associated with movements of the vertebral column.

The deep muscles develop embryo logically in the back, and are thus described as intrinsic muscles. The superficial and intermediate muscles do not develop in the back, and are classified as extrinsic muscles.

\section{Muscular Anatomy of the Back}

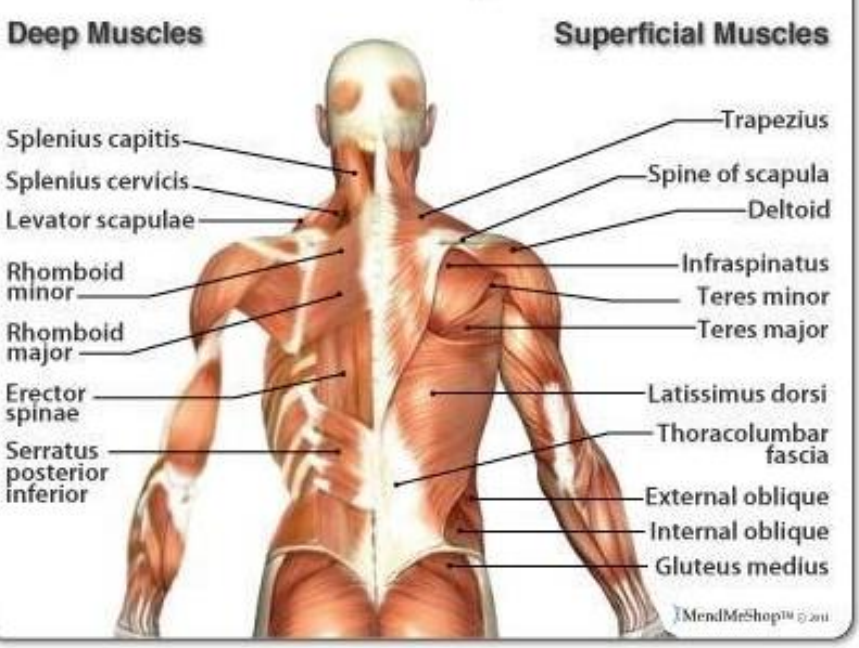

The primary back muscles are the:

\section{$>$ LatissimusDorsi}

$>$ Rhomboids

$>$ Trapezius

$>$ Posterior Deltoid

$>$ And your Erector Spinae, which supports your spine. 
Muscles of the Abdomen:-
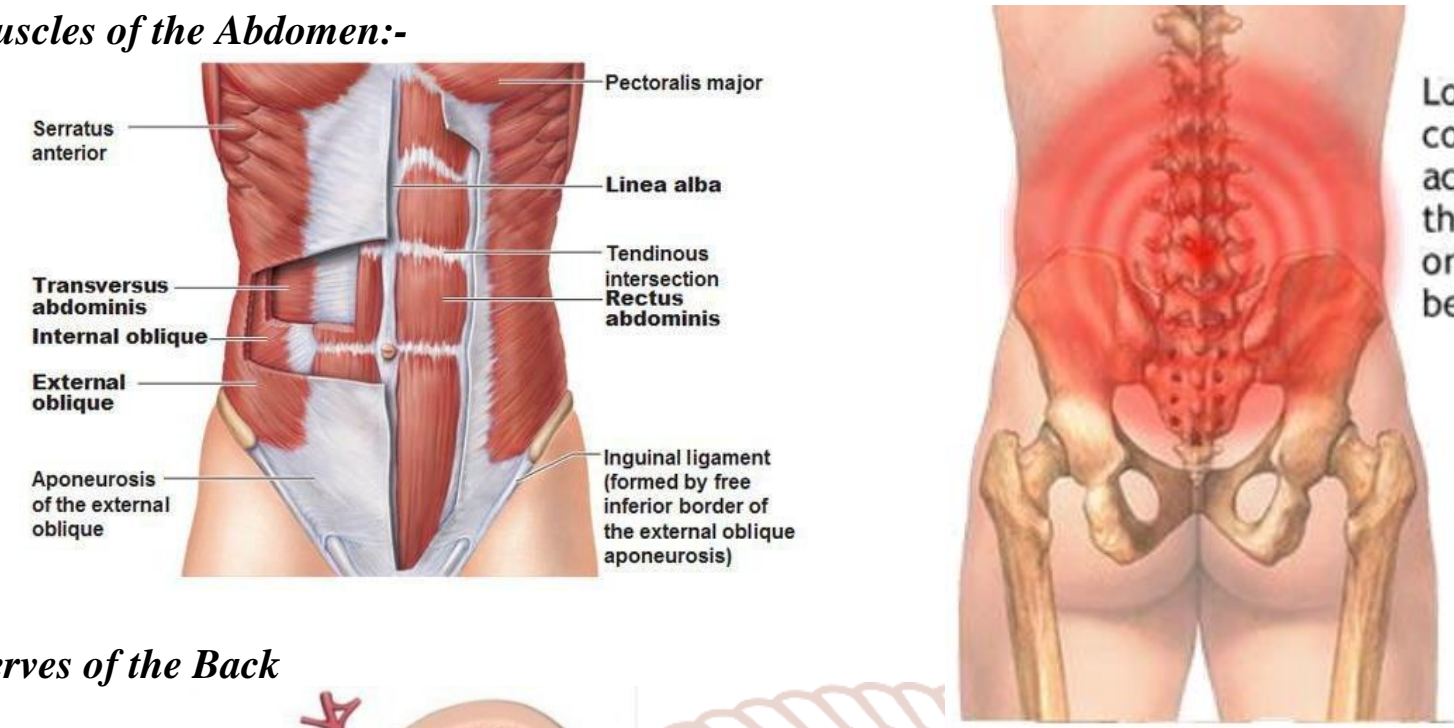

Low back pain is very common. It can be acute, lasting less than one month, or chronic, lasting beyond three months

Nerves of the Back

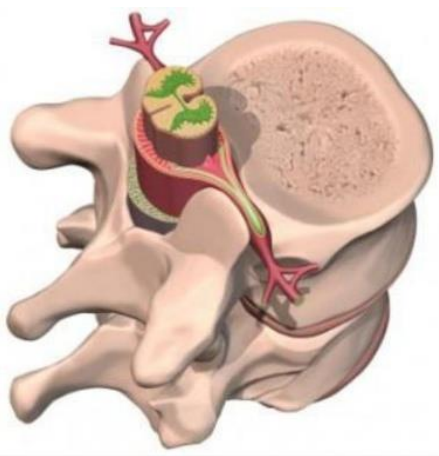

Lumbar segment, spine, nerves

\section{MECHANICAL BACK PAIN}

\section{Vascular structures of the Back}

Arteries supply the vertebrae, ligaments, and muscles with nourishment.

\section{Facet Joints in Motion}

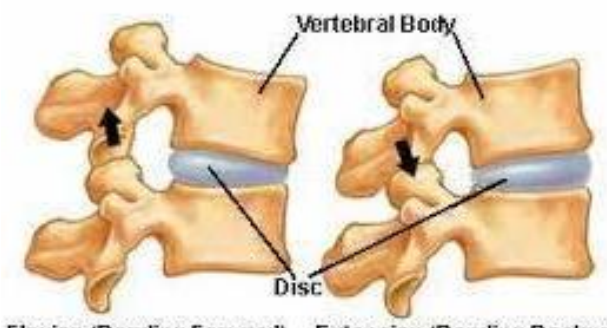

Flexion (Bending Forward) Extension (Bending Backward)

\section{- Muscle, ligament, tendon strain} including herniated disc

- Apophyseal joint arthritis

- Spinal stenosis

- Spondylolysis, spondylolisthesis

- Scoliosis
- Discogenic disorders

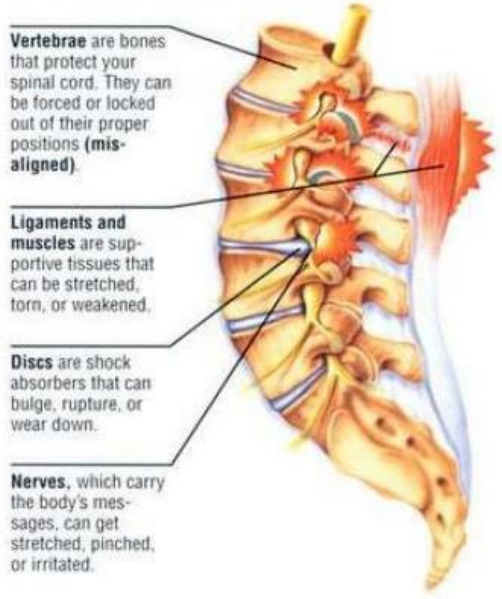

\section{INTRODUCTION:-}

Mechanical pain is the general term that refers to any type of back pain caused by placing abnormal stress and strain on muscles of the vertebral column. Typically, mechanical pain results from bad habits, such as poor posture, poorly-designed seating, and incorrect bending and lifting motions.

Mechanical pain is often called back strain because it is linked with the movement, or "the mechanics" of the spine. This type of pain occurs when injury to the spine's discs, facet joints, ligaments, or muscles results in inflammation. ... Mechanical pain can be caused by many conditions in the spine.

\section{REVIEW OF LITERATURE:-}

1. Acute mechanical back pain is a common medical problem. Acute pain is pain that has been present less than 4 to 6 weeks. Mechanical means the source of the pain may be in the spinal joints, discs, vertebrae, or soft tissues. Acute mechanical back pain may also be called acute low back pain, lumbago, idiopathic low back pain, lumbosacral strain or sprain, or lumbar syndrome.

2. Mechanical low back pain refers to back pain that arises intrinsically from the spine, intervertebral disks, or surrounding soft tissues. This includes lumbosacral muscle strain, disk herniation, lumbar spondylosis, spondylolisthesis, spondylolysis, 
vertebral compression fractures, and acute or chronic traumatic injury. Repetitive trauma and overuse are common causes of chronic mechanical low back pain, which is often secondary to workplace injury. Most patients who experience activity-limiting low back pain go on to have recurrent episodes. Chronic low back pain affects up to $23 \%$ of the population worldwide, with an estimated $24 \%$ to $80 \%$ of patients having a recurrence at one year.

3. Most people with mechanical back pain experience pain primarily in the lower back. The pain may radiate (spread) to the buttocks and thighs. Many people may also experience spasms with mechanical back pain. The symptoms of mechanical back pain are generally more noticeable with flexion of the back and when lifting heavy objects.

4. Men and women are equally affected by low back pain, which can range in intensity from a dull, constant ache to a sudden, sharp sensation that leaves the person incapacitated. Pain can begin abruptly as a result of an accident or by lifting something heavy, or it can develop over time due to age-related changes of the spine. Sedentary lifestyles also can set the stage for low back pain, especially when a weekday routine of getting too little exercise is punctuated by strenuous weekend workout.

5. Most low back pain is acute, or short term, and lasts a few days to a few weeks. It tends to resolve on its own with self-care and there is no residual loss of function. The majority of acute low back pain is mechanical in nature, meaning that there is a disruption in the way the components of the back (the spine, muscle, intervertebral discs, and nerves) fit together and move.

6. Subacute low back pain is defined as pain that lasts between 4 and 12 weeks.Chronic back pain is defined as pain that persists for 12 weeks or longer, even after an initial injury or underlying cause of acute low back pain has been treated. About 20 percent of people affected by acute low back pain develop chronic low back pain with persistent symptoms at one year. In some cases, treatment successfully relieves chronic low back pain, but in other cases pain persists despite medical and surgical treatment.
7. Low back pain is a widespread affliction involving impaired functioning of the normal mechanics of the lumbar (low back) muscles, connective tissues, ligaments and joints, often causing pain and discomfort. Low back pain's highest prevalence is postural/functional based, there may also be genetic predispositions. It ultimately involves some form of improper biomechanics. This altered function of the normal mechanics of the body affects the muscles, tendons, connective tissue, joints and ligaments of the low back resulting in pain and discomfort.

8. The majority of people will experience mechanical low-back pain sometime in their lives. Mechanical low-back pain can be defined as pain resulting from excessive or prolonged posture issues, such as too much sitting or standing. Pain can also be caused from overuse or muscle strain, or it could be pain secondary to injury or deformity relating to the spinal structures, such as bone, ligaments, discs, joints, nerves, and meninges. Mechanical back pain persisting for more than 4 to 6 weeks may require diagnostic testing and imaging. Nonetheless, treatments are available, icincluding physical therapy, with different programs specifically targeted toward different causes.

9. Mechanical back pain is most commonly the lower back, occurring as a result of strained muscles of the back. The vertebral column is supported by muscles and soft tissues. Undue stress on these muscles and soft tissues can damage the muscular support. Mechanical back pain can be short term (acute) or long lasting (chronic) in nature and may be commonly seen in young individuals.

10. Physical therapy and rehabilitation techniques help in relieving muscle strain and reducing mechanical back pain. Resuming work and performing regular activities following mechanical back pain depend on the severity of the condition. Most of the cases of back pain recover in few days or weeks and regular activities can be taken up. However, it is better to avoid strenuous physical activities, bending and lifting heavy weights if one is suffering from mechanical back pain. 


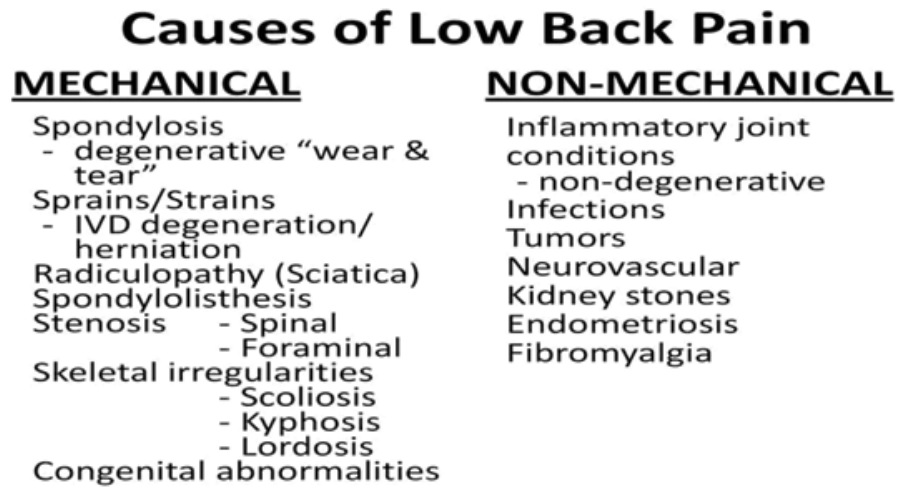

\section{Posture Pattern and Literature:-}

The posture pattern in lower body variation is often termed as "lower crossed syndrome" .

Typically including:

1. Long weak hamstrings

2. Short tight hip flexors

3. Gluteus, over arching of the low back (lordosis)

4. Tight extensors and weak long abdominals

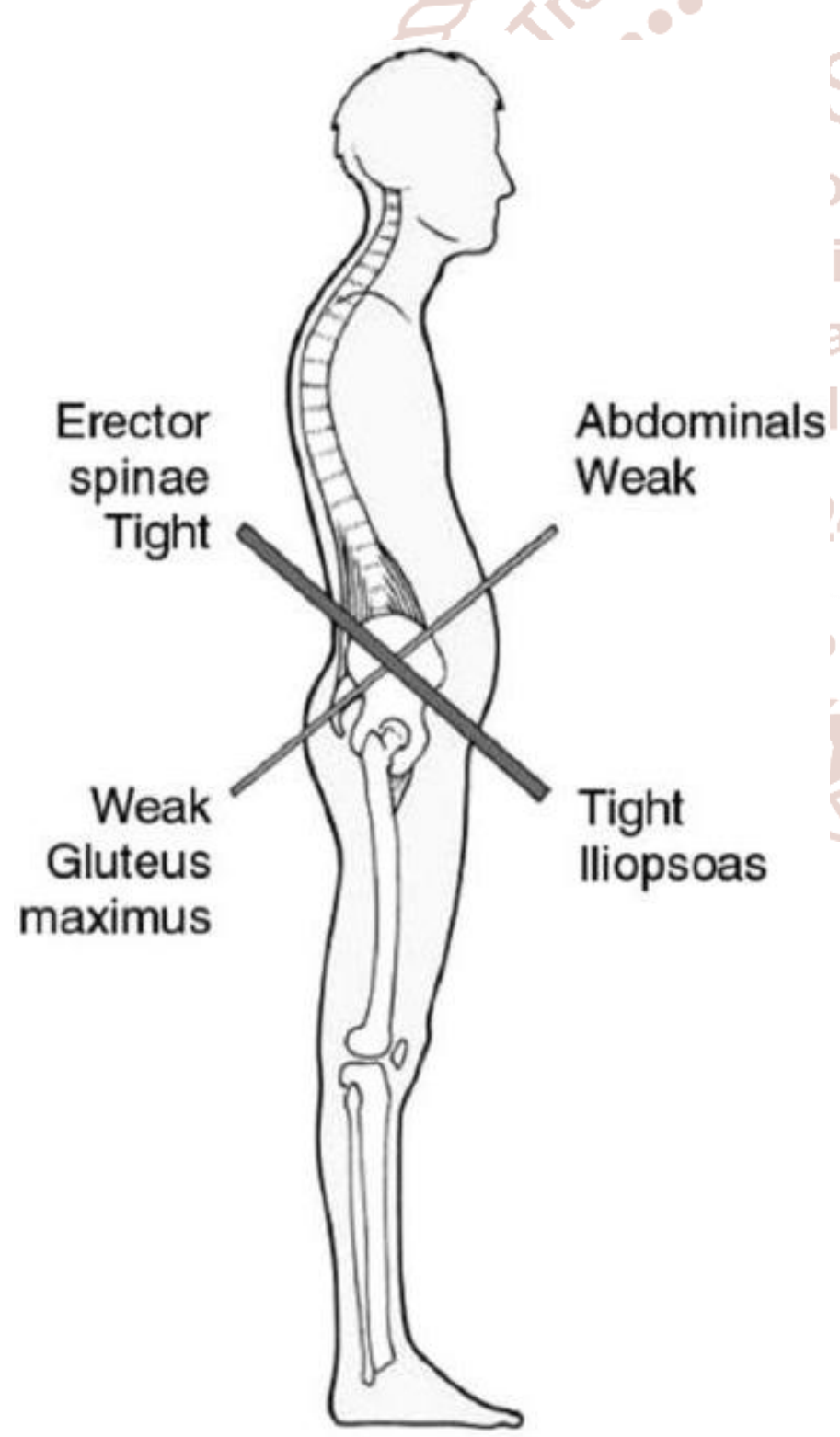

The 'Unterkreuz syndrome' is also known as pelvic crossed syndrome, lower crossed syndrome or distal crossed syndrome. The lower crossed syndrome (LCS) is the result of muscle strength imbalances in the lower segment. These imbalances can occur when muscles are constantly shortened or lengthened in relation to each other. The lower crossed syndrome is characterized by specific patterns of muscle weakness and tightness that cross between the dorsal and the ventral sides of the body. In LCS there is over activity and hence tightness of hip flexors and lumbar extensors. Along with this there is under activity and weakness of the deep abdominal muscles on the ventral side and of the gluteus maximus and medius on the dorsal side. The hamstrings are frequently found to be tight in this syndrome as well. This imbalance results in an anterior tilt of the pelvis, increased flexion of the hips, and a compensatory hyperlordosis in the lumbar spine.

These are the major reasons for mechanical LBA without significant pathology.

In this research, we'll improve the strength of back as well as abdominal muscle and correct the tone of muscle by using good ergonomics.

\section{CLASSIFICATION OF LBA:-}

Low back pain may be classified by duration as:-

$>$ Acute (Pain Lasting Less Than 6 Weeks)

$>$ Sub-Chronic (6 To 12 Weeks), Or

Chronic (more than 12 weeks).

The condition may be further classified by the underlying cause as mechanical, non-mechanical, or referred pain.

\section{Preventative Measures for Low Back Pain:}

While Standing - place a box or stool under one foot or standing on a ergonomic mat.

While Sleeping - lie on your back on a medium mattress with a pillow underneath your knees, or sleeping on your side with a pillow between your legs to minimize the stress through the hips and pelvis. Sleeping on your stomach increases the lordosis of the lumbar spine and puts excessive pressure on the low back.

While Sitting - sit straight back in chairs with lumbar support. If there is no lumbar support, place a small pillow in the small of the back to maintain the natural 
International Journal of Trend in Scientific Research and Development (IJTSRD) ISSN: 2456-6470 lumbar curve. Your feet should be supported, never hanging. You may need to lower or raise the chair and place a stool under your feet.

While Lifting - when lifting or bending below waist level:

Bend knees to reach object

Hold object close to your body

Straighten your legs to bring you to erect posture
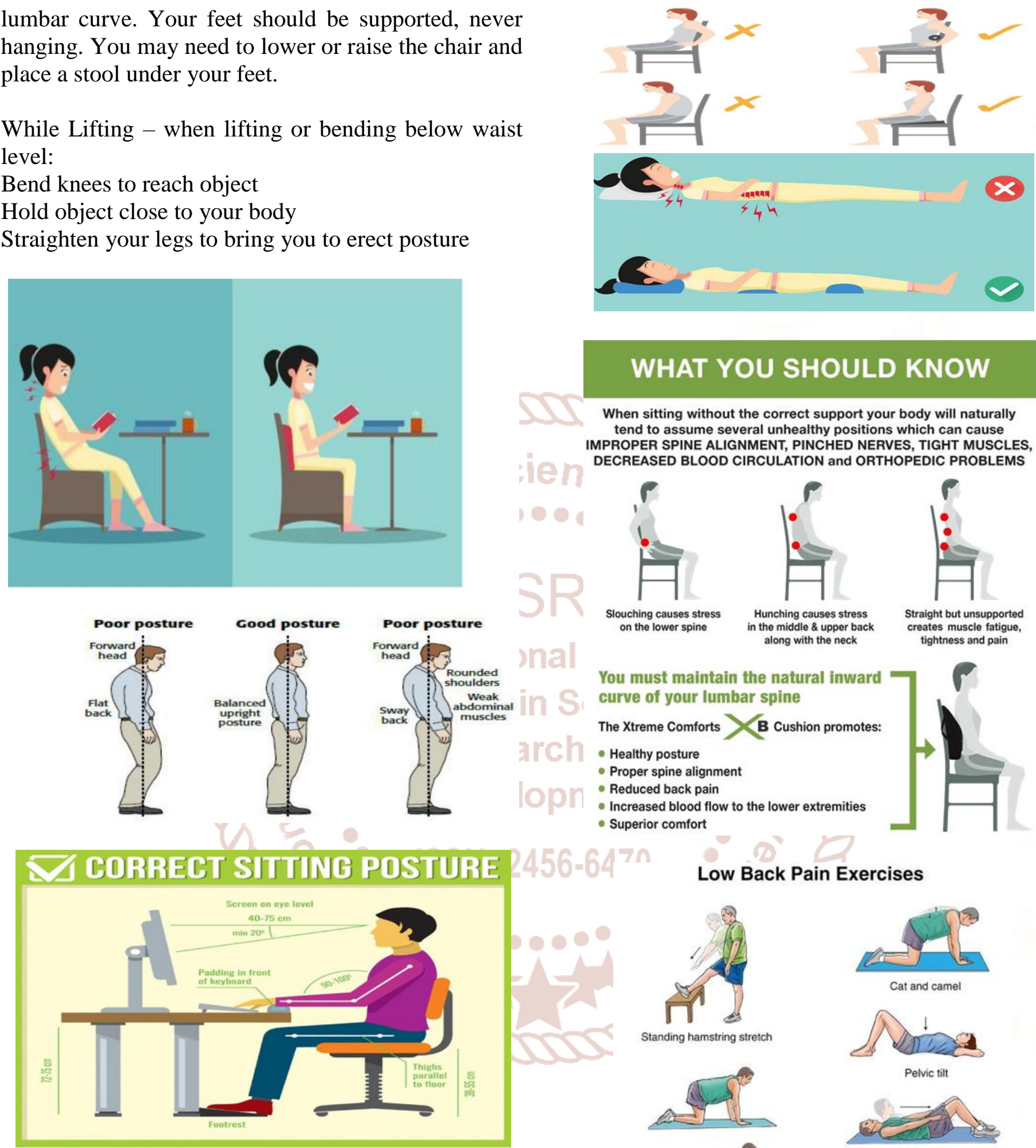

\section{WHAT YOU SHOULD KNOW}

When sitting without the correct support your body will naturally tend to assume several unhealthy positions which can cause IMPROPER SPINE ALIGNMENT, PINCHED NERVES, TIGHT MUSCLES, DECREASED BLOOD CIRCULATION and ORTHOPEDIC PROBLEMS

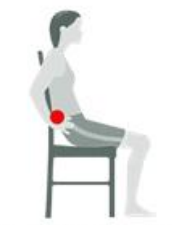

Slouching causes stress on the lower spine

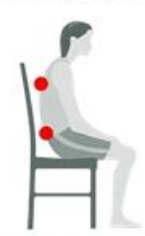

Hunching causes stress the middle \& upper back along with the neck

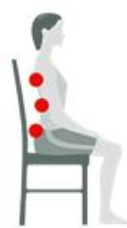

Straight but unsupported creates muscle fatigue, tightness and pain

You must maintain the natural inward curve of your lumbar spine

The Xtreme Comforts B Cushion promotes:

- Healthy posture

- Proper spine alignment

- Reduced back pain

- Increased blood flow to the lower extremities

- Superior comfort

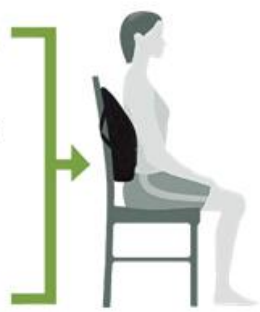

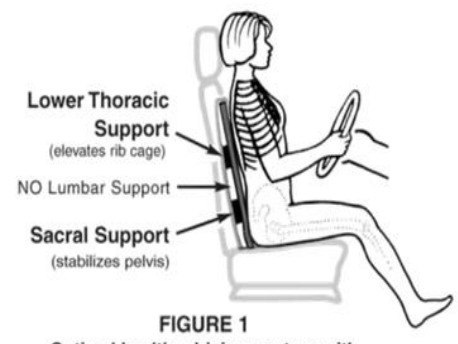

Optimal healthy driving posture with sacral and lower thoracic supports, but no lumbar support.

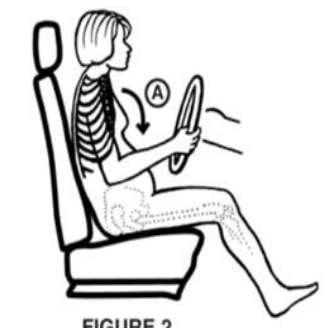

FIGURE 2

Typical slumped driving posture due to

lowering of rib cage (A)

without lower thoracic support.

\section{Low Back Pain Exercises}

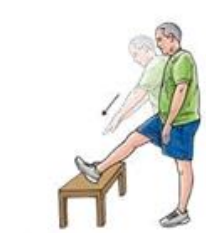

Standing hamstring stretch
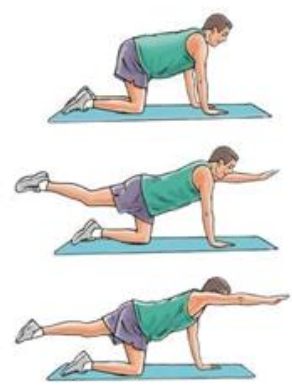

Quadruped arm/leg raise

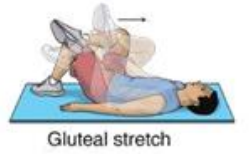

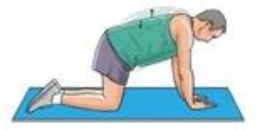

Cat and camel

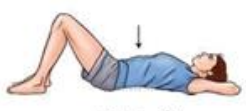

Pelvic tilt

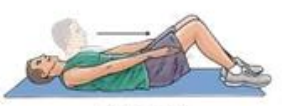

Partial curl
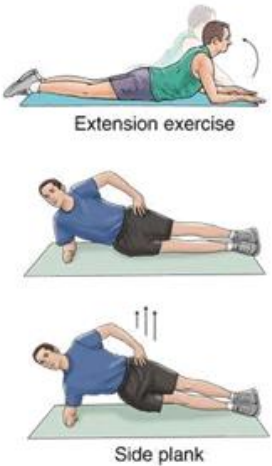
International Journal of Trend in Scientific Research and Development (IJTSRD) ISSN: 2456-6470

OFFICE EXERCISES:-
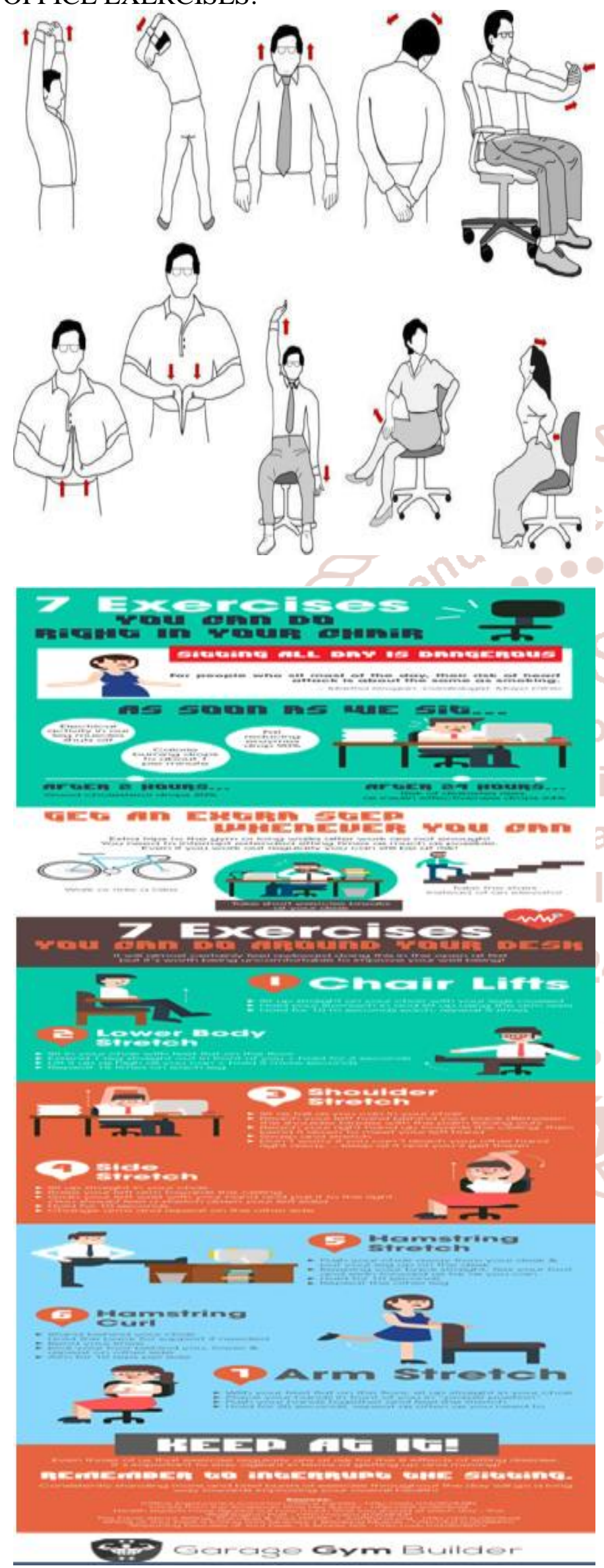



\section{QUESTIONNAIRE:-}

General questions:-

$>$ Name -

$>$ Your age- (in yrs.)

$>$ Your gender-

> Your weight- (in kgs)

$>$ Your height- (in inches)

$>$ Occupation-

1) - How do you wake-up everyday?

1. Fresh mood 2. Lethargic mood 3. Sad mood

2) How many hours do you sleep a day?
1. 4 hrs.
2. 6-7 hrs
3. 8-9 hrs

3) How do you travel to your workplace? 1. Walk 2.2 wheeler 3.4 wheeler

4) What kind of work do you usually have?

1. Prolonged sitting 2. Walking 3. Standing

5) How is your physical body fitness?

1. Good 2. 2 wheeler 3. 4 wheeler

6) What do you need at your work?

1. Meditation 2. Strength 3.Endurance

7) How you feel at the end of the day?
1. Active
2. Tired
3. Lazy

8) Any kind of morning stiffness when you get up?
1. Yes
2. No
3. Sometimes 
9) Do you follow any kind of physical exercise regime?
1. Yes
2. No
3. Sometimes

10) Do you pick any heavy object while working?
1. Yes
2. No
3. Sometimes

11) Do you follow proper ergonomics in your workstation?
1. Yes
2. No
3. Sometimes

12) What kind of foot wear you use during work time?
1. Flats
2. Heels
3. Sports shoes

13) Do you stretch your arms \& back while in work?
1. Yes
2. No.
3 sometimes

14) What kind of posture you always sit?
1. Bend
2 .straight
3. Straight-forward

15) Do you suffer from any kind of backache problem?
1. Yes
2. No
3. Some times

\section{ETIOLOGY AND RISK FACTORS}

Since, in the majority of cases etiology is multifactorial, identifying a single cause or even the principal cause of low back pain is an extremely difficult task. Both individual and professional risk factors are involved in the genesis of low back pain. The most common individual risk factors are: age, sex, body mass index, muscle imbalances, muscle strength, socioeconomic conditions and the presence of other infirmities. The most common professional risk factors involve incorrect movements and postures caused by inadequate working environments, the functions of available equipment and the ways in which work is organized and carried out.

\section{DIAGNOSIS:-}

The elevate incidence of abnormal findings in imaging exams conducted on asymptomatic people mean that it is imperative to correlate these findings with information from patient history and physical examinations. A diagnosis of occupational low back pain also demands detailed occupational history taking and careful analysis of the way work is organized and of the environment in which it takes place.

It is not enough to simply wait for the patients to mention their complaints. They should be actively elicited. The physician must avoid adopting a posture of directing questioning exclusively at symptoms located in the lumbar region and expand the arsenal of questions in order to detect with greater precision the true origin of low back pain and characterize the nonorganic symptoms that are often present, without being led by the appearance of imaging exams.

Sudden "loss of strength" of a muscle of group of muscles (instantaneous refusal at a certain point during a maneuver requiring strength) is behavior that is characteristic of non-organic pain. Diseases that cause muscle weakness manifest during physical examination with a consistent degree of loss of strength. This loss of strength (smooth and constant) is almost impossible to simulate for people with nonorganic symptoms.

Patients who claim to be unable to work because they are lame, should have signs of uneven shoe sole wear. Symmetrically worn soles (in the absence of heel deviations) may be a sign that the limping or claudication is non-organic. Using questionable or unprescribed or theses, including walking sticks and kidney belts, is another sign associated with nonorganic complaints, especially when there is no corresponding atrophy or cutaneous signs of prolonged use.

Checking for calluses on the hands may verify whether a worker is indeed leaving off physical activities. Lacerated hands and dirt under the nails are also useful signs. An absence of muscle atrophy after a prolonged period of inactivity and maintenance of muscle tone of the trunk and pectoral girdle are indications of non-organic pain.

Mechanical back pain needs to be thoroughly evaluated taking into consideration the medical history and the presenting complaints. A detailed history or injuries, accidents, surgeries and history of any medical disorders needs to be taken. Clinical examination of the painful back region may reveal tenderness in the area. Mechanical back pain, as against the inflammatory back pain may have no signs of inflammation.

Investigations like $\mathrm{X}$-rays and MRI may be done to identify fractures, changes in the bone and signs of injury, muscle or ligament strain and nerve injury. If there are other associated symptoms, which need to be ruled out, additional tests may be ordered. 


\section{PROGNOSIS:-}

The occupational prognosis of patients with chronic low back pain should not be based on the appearance of images (degeneration of discs or osteophytes) of the lumbar spine of the patient or compensation seeker. Detection of signs suggestive of non-organic pain does not imply poor prognosis - if patients are treated properly. The presence of unfavorable psychosocial factors can predict a greater number of days off work, if the sufferer is not treated correctly. One group of researchers has proven that even patients with apparently refractory chronic low back pain strongly associated with psychosocial factors may exhibit significant improvements, if managed with multidisciplinary rehabilitation that adequately deals with the somatic symptoms and not just the regional physical pains or with supposed anatomic findings that are of little relevance. Another study supported these conclusions that treatment and improvement is possible with patients with chronic low back pain, even in long-term cases.

\section{PREVENTION}

Prevention of occupational low back pain involves physical, organizational and cognitive measures. The physical measures should deal with the biomechanical aspects, posture at work, handling of material and loads, repetitive movements, job descriptions and occupational health and safety. Organizational prevention should focus on communication, on resource management, on job descriptions, on the organization of time at work, on teamwork, on paradigms of work, on cooperative working, organizational culture, organizational networking, teleworking and quality management. The cognitive component involves studying psychological processes, mental workload at work, decision making, specialist performance, man-machine interaction, stress and training. In this way, the psychosocial factors that contribute to the emergence of occupational low back pain can be prevented, including job dissatisfaction, monotonous work and the wear provoked by work overload, by the lack of autonomy and by competition with colleagues.

\section{TREATMENT:-}

Treatment of mechanical back pain usually includes rest for a specified period along with pain relieving medicines and muscle relaxants. Bed rest is usually recommended only for few days, till the muscle spasms and severe pain subsides.
Ice pack or cold compresses may be advised during acute phase of mechanical back pain to relieve muscular strain. Warm compresses may be recommended to relax tensed muscles. Alternate cold and warm compresses also help to provide relief in mechanical back pain and should be practiced as advised by the physician.

Physical Therapy for Mechanical Back Pain: PT and movement may be advised after the initial pain reduces. Physical activity promotes better healing of the strained muscles and improves blood circulation. Physical therapy and rehabilitation techniques help in relieving muscle strain and reducing mechanical back pain.

Resuming work and performing regular activities following mechanical back pain depend on the severity of the condition. Most of the cases of back pain recover in few days or weeks and regular activities can be taken up. However, it is better to avoid strenuous physical activities, bending and lifting heavy weights if one is suffering from mechanical back pain. It is advisable to seek medical opinion before involving in sports, recreational activities and adventure games.

Further episodes of back pain can be prevented by following a healthy routine, regular physical exercises and a healthy diet. Avoid smoking, limit alcohol, drink more water and maintain weight to remain healthy.

\section{Exercises for Mechanical Back Pain}

Exercises for mechanical back pain should be performed under supervision and as advised by the physician.

Find some of the best back strengthening exercises that can be helpful for mechanical back pain.

Lie on back with knees raised and feet on the floor and perform the following:

> Static Back Exercise - Press the low back region, so that it touches the floor; hold and release.

$>$ Pelvic Bridging - Raise your hip and pelvic portion, hold and release.

> Knee to chest - Bend one knee and bring it close to touch the chest. Repeat the same with other knee. Perform the same with both knees together.

$>$ Cat-Cow Stretch - Lie on all fours. Tighten the back to form a curve of the back and then release. 
Walking in swimming pool and aquatic exercises also help in relieving mechanical back pain and should be performed as advised.

\section{RESULT:-}

By studying the questionnaire and the different age groups before and after physiotherapy treatment of mechanical Low backache, their lower back and abdominal muscles we found that "Correction of their posture and ergonomics decreases the mechanical low backache."

The graph below will help us to understand the comparison of age, posture, severity of pain and ergonomics.

\section{SAMPLE SIZE- 30}

AGE GROUP: - (20-30 Yrs., 30-40 yrs. , 40-50 yrs. , 50-60 yrs.)

Before physiotherapy treatment:-

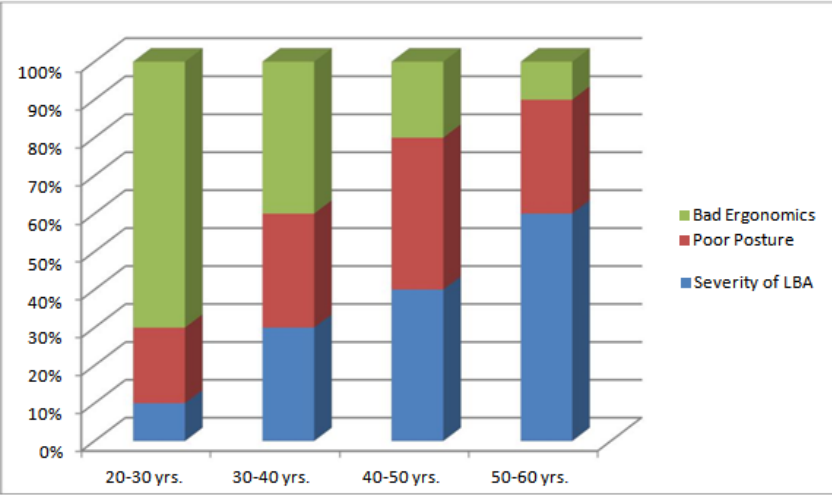

After Physiotherapy treatment graph will be like;

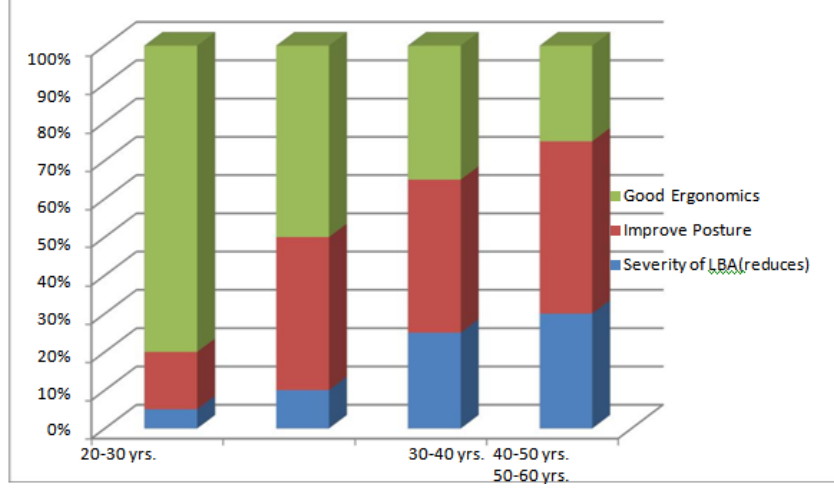

The Pain Scale is described below:0-10 NUMERIC PAIN RATING SCALE

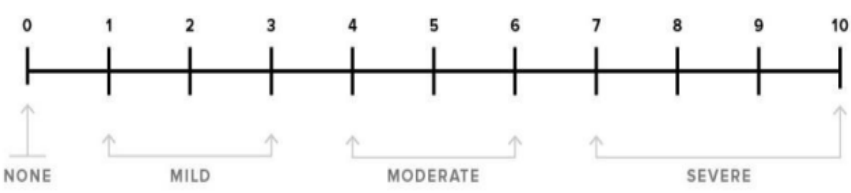

Manual Muscle Testing Grading System:-

\begin{tabular}{|c|c|c|c|}
\hline 0 & 0 & & No visible or palpable contraction \\
\hline Trace & I & 1 & Visible or palpable contraction (No ROM) \\
\hline Poor & & & Partial ROM, gravity eliminated \\
\hline Poor & II & 2 & Full ROM, gravity eliminated \\
\hline Poor ${ }^{+}$ & & $2^{+}$ & $\begin{array}{l}\text { Gravity eliminated/slight resistance or }< \\
1 / 2 \text { range } \\
\text { against gravity }\end{array}$ \\
\hline Fair & & & $\begin{array}{l}>1 / 2 \text { but }<\text { Full ROM, against } \\
\text { gravity }\end{array}$ \\
\hline Fair & III & & Full ROM against gravity \\
\hline Fair ${ }^{+}$ & & & $\begin{array}{l}\text { Full ROM against gravity, slight } \\
\text { resistance }\end{array}$ \\
\hline Good- & & $4^{-}$ & Full ROM against gravity, mild resistance \\
\hline Good & & IV & 4 Full ROM against gravity, moderate resistance \\
\hline Good+ & & $4+1$ & Full ROM against gravity, almost full resistance \\
\hline Normal & & V I & Normal, maximal resistance \\
\hline
\end{tabular}

\section{CONCLUSION:-}

Following any period of prolonged inactivity, a regimen of low-impact exercises is advised. Speed walking, swimming, or stationary bike riding 30 minutes daily can increase muscle strength and flexibility. Yoga also can help stretch and strengthen muscles and improve posture. Consult a physician for a list of low-impact, age-appropriate exercises that are specifically targeted to strengthening lower back and abdominal muscles.

Always stretch before exercise or other strenuous physical activity.

Don't slouch when standing or sitting. The lower back can support a person's weight most easily when the curvature is reduced. When standing, keep your weight balanced on your feet.

$>$ At home or work, make sure work surfaces are at a comfortable height.

Sit in a chair with good lumbar support and proper position and height for the task. Keep shoulders back. Switch sitting positions often and periodically walk around the office or gently stretch muscles to relieve tension. A pillow or rolled-up towel placed behind the small of the back can provide some lumbar support. During prolonged periods of sitting, elevate feet on a low stool or a stack of books.

Wear comfortable, low-heeled shoes. 
Sleeping on one's side with the knees drawn up in a fetal position can help open up the joints in the spine and relieve pressure by reducing the curvature of the spine. Always sleep on a firm surface.

$>$ Don't try to lift objects that are too heavy. Lift from the knees, pull the stomach muscles in, and keep the head down and in line with a straight back. When lifting, keep objects close to the body. Do not twist when lifting.

$>$ Maintain proper nutrition and diet to reduce and prevent excessive weight gain, especially weight around the waistline that taxes lower back muscles. A diet with sufficient daily intake of calcium, phosphorus, and vitamin D helps to promote new bone growth.

$>$ Quit smoking. Smoking reduces blood flow to the lower spine, which can contribute to spinal disc degeneration. Smoking also increases the risk of osteoporosis and impedes healing. Coughing due to heavy smoking also may cause back pain.

In a few cases, physical therapy may be an essential part of acute back pain rehabilitation. It is important that you work with a physical therapist trained in the exercise approach to promote rapid healing.
Active physical therapy can help shorten recovery time and return you to work and leisure activities as quickly as possible. Active physical therapy is an exercise program that may require home exercises as frequently as every 2 hours while you are awake. The exercises generally take about 5 minutes to do and do not require special equipment, nor do you need to go to a gym to do them.

\section{REFERENCES}

1. https://my.clevelandclinic.org/health/diseases/487 9-acute-mechanical-back-pain

2. https://www.aafp.org/afp/2018/1001/p421.html

3. https://my.clevelandclinic.org

4. www.ninds.nih.gov

5. https://www.ninds.nih.gov

6. https://www.ninds.nih.gov

7. https://leggehealth.ca/portfolio-item/low-backpain/

8. https://www.sharecare.com/health/backpain/what-mechanical-low-back-pain

9. https://www.epainassist.com/backpain/mechanical-back-pain

10. www.epainassist.com 